\title{
Von Sorgearbeit und gesellschaftlichen Scheinwerfern: \\ Die Home-Allround-Mum als ambivalente Sozialfigur der Corona-Pandemie
}

Erschienen in: Sozialfiguren der Corona-Pandemie

Von: Lilian Hümmler, Sarah Speck

Mit schmerzverzerrtem Gesicht, vermutlich überarbeitet und von Kopfschmerzen geplagt, stützt eine junge Frau den Kopf in die Hände. Sie sitzt, das Smartphone am Ohr, in der Küche vor Laptop, Headset und einem Berg Unterlagen. Die Augen flirren wahrscheinlich noch von der letzten Videokonferenz, die mal wieder länger dauerte als geplant. Länger ist auch die To-Do-Liste geworden, welche die Spitze des Zettelbergs bildet. Und jetzt ist da noch die Kollegin am Telefon, die „nur mal kurz" das Meeting nachbesprechen wollte. Flurgespräch ist nicht mehr - Emo-Talk also am privaten Handy. Im Hintergrund stapft ein lachendes Schulkind mit buntem Musikgerät durchs Bild und wir hören schon förmlich die Kinderlieder. Eigentlich müsste doch schon längst das Mittagessen auf dem Herd stehen, aber der Kollegin geht es wirklich schlecht, die Schulaufgaben des Kindes sind bestimmt noch nicht erledigt und der Chef wollte doch schnell die eine Mail bekommen. Aber wie soll hier zwischen Mittagessen, Kinderbetreuung und -beschulung überhaupt konzentriertes Arbeiten funktionieren? Die spezifische Kamera-Einstellung auf das Geschehen dieses sehr spezifischen, aber dennoch für viele sich ähnlich darstellenden Alltags, ist vergleichbar mit einem gesellschaftlichen Scheinwerfer, der neue Sichtbarkeiten für zum Teil alte Phänomene schafft. Dass das Home-Office in Corona-Zeiten die Idee der Vereinbarkeit von Beruf und Familie ad absurdum führt, zeigen auch die Berichte überforderter Eltern in sozialen Netzwerken: Wie soll auch noch Home-Schooling in den eigenen Arbeitstag integriert werden? Auf welche Weise können Kinder während der Telefonkonferenz beschäftigt werden, um eben nicht als S piderman verkleidet durchs Bild zu springen? Zahlreiche Blogbeiträge versuchen auf diese Fragen überforderter Eltern und vor allem Mütter zu reagieren, liefern Tipps und Tricks, unter ihnen allen voran der Appell: „Sei flexibel und reagiere auf die Situation“. Dieser Diskurs rund um die Regulierungen zur Eindämmung der Corona-Pandemie und insbesondere die Schließung der Kitas und Schulen im Frühjahr 2020 markierte die Geburtsstunde einer neuen Sozialfigur - der HomeAllround-Mum. Sie charakterisiert die Mutter, die im Home-Office und im Home-Office arbeitet sowie das Home-Schooling der Kinder übernimmt. Konkret erbringt sie folglich ihre Erwerbsarbeit zu Hause, kümmert sich um Kinderbetreuung und -beschulung, übernimmt mitunter die Pflege von alten und/oder erkrankten Angehörigen und stemmt 
den Haushalt, also Putzen, Einkaufen, Kochen, Abwaschen, Müll wegbringen, Wäsche waschen und Aufräumen. Wir sind ihr nicht nur in medialen Repräsentationen, sondern auch im Rahmen einer qualitativen, explorativen Studie zur ,Neuordnung des Privaten“ begegnet. ${ }^{1}$ Die folgenden Ausführungen basieren auf ersten Ergebnissen.

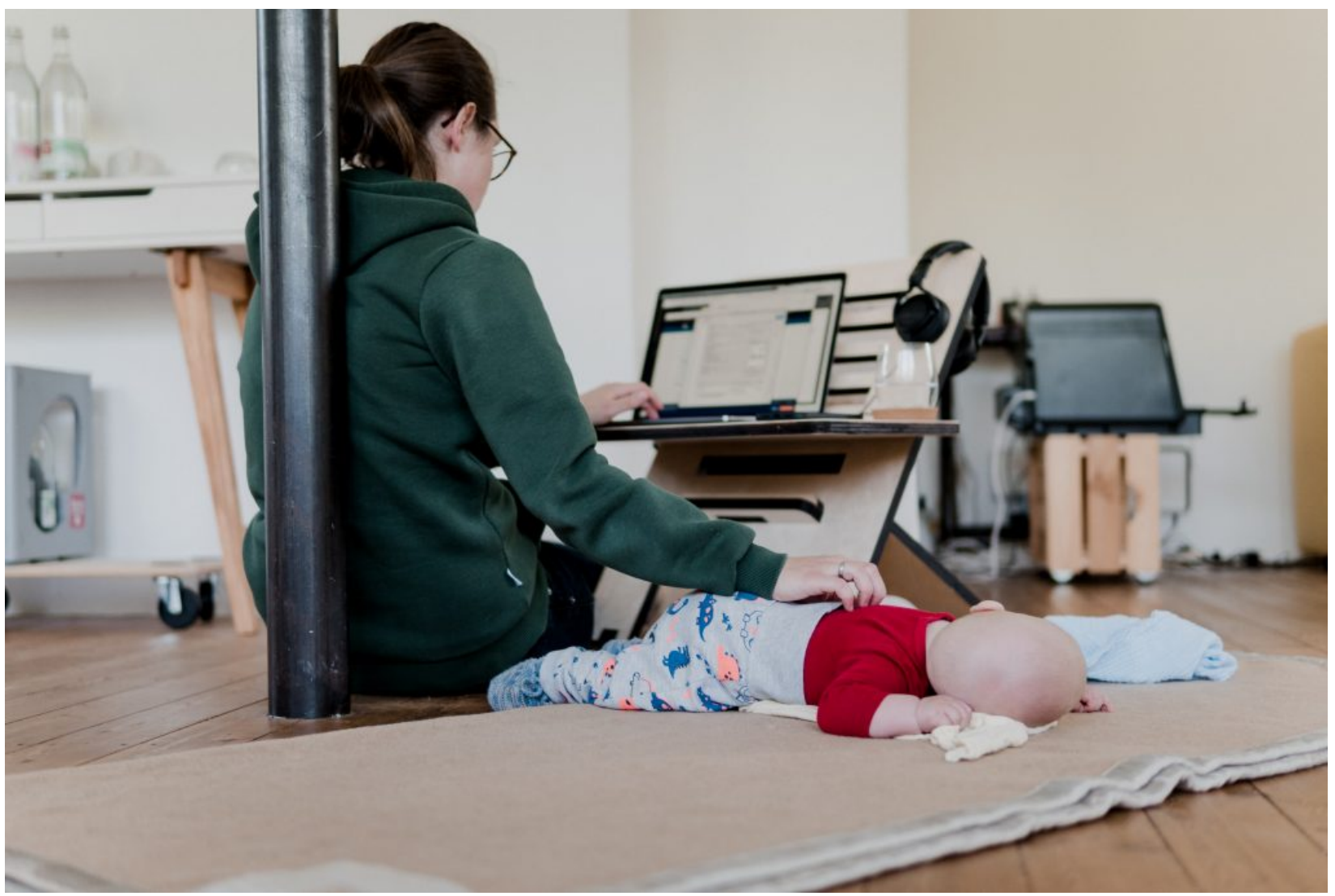

Mother working from home - at flexible and height-adjustable desk with baby. Photo by $\underline{\text { Standsome }}$ Worklifestyle on Unsplash.

Mit der Home-Allround-Mum und der Diskussion über die Auswirkungen der politischen Maßnahmen gelangte häusliche Sorgearbeit, die weiterhin ebenso vergeschlechtlicht wie gesellschaftlich unsichtbar ist, in den Blickpunkt medialer und politischer Debatten. Ähnlich wie die plötzliche Aufmerksamkeit für ,systemrelevante Berufe‘ wurde im Zuge der Pandemie deutlich, dass in unserer weiterhin auf einer stabilen

Geschlechterordnung basierenden, kapitalistischen Gesellschaft Arbeiten existieren, die nicht oder nur schlecht entlohnt werden und dennoch unabdingbar für das (gesellschaftliche) Leben sind. Diese feminisierten Tätigkeiten - ob im Krankenhaus, an der Supermarktkasse oder eben Zuhause - werden entlang von Geschlecht, aber auch entlang von Klasse und rassistischer Segregation verteilt. Mit Blick auf die private Sorgearbeit materialisierte sich die neue gesellschaftliche Aufmerksamkeit dann im sogenannten ,Kinderbonus', einer einkommensunabhängigen Einmalzahlung von $300 €$ pro Kind, und führte dazu, dass im politischen Diskurs die Schließung von Kitas und Schulen heute, mitten in der zweiten Corona-Welle, als das allerletzte probate Mittel erscheint. $^{2}$

Lässt sich diese Entwicklung nun als Erfolg im Sinne der Gleichberechtigung verbuchen? Wurde die feministische Forderung nach gesellschaftlicher Anerkennung von Sorge- und Reproduktionsarbeit im Zuge der Corona-Pandemie eingelöst? Wie 
immer in der Wissenschaft ist es komplizierter: Anhand der Sozialfigur der HomeAllround-Mum lassen sich die Ambivalenzen aufzeigen, die ihre Entstehung erst ermöglichten.

Zunächst blieb die Diskussion der mit Sorgearbeit verbundenen Fragen, sowohl im Privaten als auch mit Blick auf die ,systemrelevanten Berufe', nur temporär. Schon über den Sommer wurde deutlich, dass der Höhepunkt medialer Sichtbarkeit bereits erreicht war und über diese Sondersituation hinaus jene im Stillen verrichteten Arbeiten, welche die Grundlage unserer Gesellschaftsordnung bilden und unser Wirtschaften überhaupt ermöglichen, gesellschaftlich gerade nicht mehr verhandelt wurden. Auch unterstreicht die durch die Alltagsnotwendigkeiten des spezifisch pandemischen Settings hervortretende Sozialfigur der Home-Allround-Mum, die eben eine Mum ist, zwar das gesellschaftliche Bewusstsein über die geschlechtliche Zuweisung von Sorgetätigkeiten - eine grundsätzliche Infragestellung dieser Geschlechterordnung erfolgte allerdings nicht. Vielmehr wurden Geschlechtererwartungen allein schon auf der bildlichen Ebene erneut normalisiert und naturalisiert: So ist es nun einmal - die Frauen machen's halt. Die oben zitierte Aufforderung zur Flexibilität in der Corona-Pandemie bedeutete in den meisten heterosexuellen Familienkonstellationen die Reduzierung der Erwerbsarbeit und insgesamt eine größere Belastung der Mütter. 

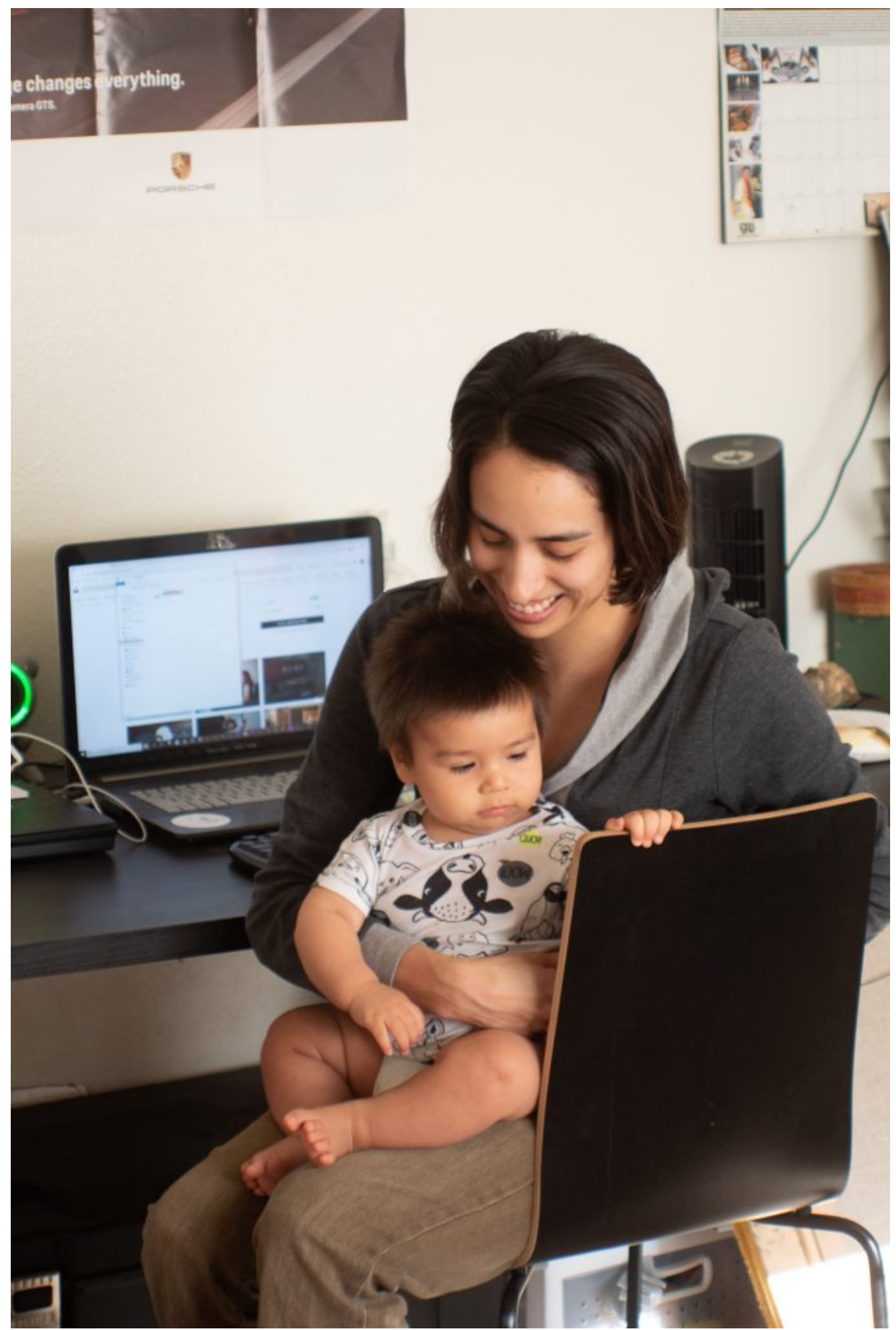

Work From Home - at desk with baby. Photo by Brian Wangenheim on Unsplash. 
Zwar ist die Home-Allround-Mum aus der gesellschaftlichen Unsichtbarkeit hervorgetreten, doch wurden durch ihre Anrufung gleichzeitig andere Lebensrealitäten unsichtbar gemacht. Als Sozialfigur scheint diese Mutter überhaupt erst auf, weil bestimmte Voraussetzungen dafür bestehen, dass sie Lohnarbeit, Kinderbetreuung und Pflege, Hausarbeit sowie Home-Schooling im häuslichen Rahmen zu bewältigen versucht. Ins ,Büro Zuhause‘ können nämlich bei Weitem nicht alle Berufe verlegt werden: Weder können Kellner*innen digital Bier ausschenken oder Saisonarbeiter*innen über Zoom Spargel ernten, noch Pflegekräfte per Online-Cloud Menschen beim Waschen unterstützen. Home-Office ist maßgeblich Berufen der Mittelklasse vorbehalten. Wer körperliche, pflegerische, soziale oder andere Dienstleistungstätigkeiten vor Ort ausübt, muss dieser Arbeit entweder unter einer vergrößerten Belastung und Ansteckungsgefahr, wie im Care-Sektor, weiterhin nachgehen, oder mit der existenziellen Sorge leben, die Kurzarbeit und Arbeitslosigkeit mit sich bringen. Auch braucht die Home-Allround-Mum überhaupt eine Erwerbsarbeit, die sie ins Home-Office verlegen kann. Erwerbslose Mütter mit Sorgeverpflichtungen, die sich angesichts schon existenter finanzieller Engpässe durch Hamsterkäufe im Lockdown noch schwieriger gestalten, werden durch diese neue Sozialfigur nicht erfasst.

Die Figur der Home-Allround-Mum ist nicht nur cis-weiblich ${ }^{3}$ vergeschlechtlicht, sondern auch heterosexuell konstruiert - Re-Traditionalisierung geht eben nur, wo es mal Tradition gab, und diese wird gesellschaftlich meist weder queer noch trans* oder nonbinär imaginiert. Schon beim gemeinsam wohnenden lesbischen Paar mit Kind gerät die Sozialfigur an ihre Grenzen: Inkorporierte Muster, die Frauen in Krisensituationen die Verantwortung für Sorgearbeiten und das Aufrechterhalten des Alltags zuweisen, werden hier obsolet. Auch die Realität der Patchwork-Familie, die mehr als zwei Elternteile umfasst und Kinderbetreuung in (räumlichen) Wechselmodellen vollzieht, wird von der Home-Allround-Mum nicht eingefangen, geschweige denn in Vorstellungen von Ausgangsbeschränkungen, die primär Kontakt mit Menschen desselben Haushalts vorsehen. Die Home-Allround-Mum verkörpert und unterstreicht das gesellschaftlich dominante Familienmodell der heteronormativen Kleinfamilie.

Sowohl die illegalisiert lebende Migrantin, die trotz erhöhtem Gesundheitsrisiko dazu gezwungen ist, weiterhin die Haushalte wohlhabender Menschen zu putzen, als auch die Rentnerin, die alle Kids im Haus betreut, damit das Home-Office der Eltern einigermaßen funktionieren kann, der*die in der Notbetreuung arbeitende Erzieher*in im Transitionsprozess sowie der alleinerziehende Vater, der eine besondere Gefährdung seines Kindes mit Atemwegserkrankungen fürchtet - all diese Menschen sorgen Tag für Tag dafür, dass (gesellschaftliches) Leben weiterhin existieren kann. All diese Menschen leisten Tag für Tag Care-Arbeit, also nicht nur ,system-' sondern vor allem ,lebensrelevante' Arbeit. Und all diese Menschen stehen im Schatten des Scheinwerferlichtes, das gesellschaftlich auf eben nur eine sehr spezifische Lebensform gerichtet wird. 
Bei aller Kritik der Ausblendungen sollte es nun aber nicht um das diskursive Ausknipsen des Scheinwerferlichts gehen - im Gegenteil: Es braucht mehr und dauerhafte gesellschaftliche Aufmerksamkeit für die verschiedenen Formen der Arbeit „zu Hause' und für die feministisch-marxistischen Perspektiven, die das Zuhause seit jeher als Labor für Ausbeutung im patriarchalen Kapitalismus analysieren. Ebenso wie für die gesellschaftliche Bandbreite an Arbeitsverhältnissen und Lebensrealitäten, die sich eben nicht nur über Home-Office und Mittelklasse erstreckt, bedarf es gesellschaftlicher Scheinwerfer, welche die Vielfalt von Geschlechter-, Familien-, Haushalts- und Sorgekonstellationen abbilden. Geschlechtertheoretische, arbeitssoziologische und intersektionale Perspektiven erlauben im Zuge dessen einen kritischen Blick auf derzeitige Entwicklungen, die auch und gerade in Krisenzeiten Aufschluss über den gegenwärtigen Zustand der Gesellschaft ermöglichen.

\section{References}

1. Von Ende März bis Anfang Mai haben wir gemeinsam mit Bea Ricke ausführliche leitfadengestützte Interviews mit Personen aus 33 Haushalten geführt. Die Auswahl der Interviewpartner*innen folgte dem Prinzip der maximalen Kontrastierung, das Sample beinhaltet unterschiedliche Berufsgruppen, darunter auch Menschen in ,systemrelevanten' Berufen, in Kurzarbeit, ohne Erwerb, sowie Personen mit und ohne Aufenthaltsstatus. Durch die leitende Frage der Studie nach der ,Neuordnung des Privaten', die auch die Re-Organisation der Arbeitsteilung angesichts der vielen anfallenden Aufgaben und der starken räumlichen Nähe beinhaltet, haben wir insbesondere Familien und mehrköpfige Haushalte befragt. Es wurden jedoch auch Interviews mit alleine wohnenden Menschen geführt.

2. Das Zuhause lässt sich im Kontext der Pandemie und insbesondere aus geschlechtertheoretischer Sicht nicht ohne die eklatante Zunahme sexualisierter und sogenannter häuslicher Gewalt betrachten, die insbesondere feministische Gruppierungen, Verbände in der Kinder- und Jugendhilfe sowie Beratungsstellen für Betroffene von Gewalt und Frauenhäuser im Frühjahr 2020 attestierten und für die Zukunft prognostizieren. Über die Ambivalenzen medialer Aufmerksamkeiten und gesellschaftliche Gewaltstrukturen schreiben Lilian Hümmler und Marilena de Andrade an anderer Stelle (

3. Cis ist eine lateinische Vorsilbe, die „diesseits“ bedeutet und als Unterscheidung zu trans (,jenseits“) dient. Cis-weiblich meint dann, dass einer Person bei Geburt das Geschlecht weiblich zugewiesen wurde und sie sich damit auch identifiziert.

SUGGESTED CITATION: Hümmler, Lilian; Speck, Sarah: Von Sorgearbeit und gesellschaftlichen Scheinwerfern: Die Home-Allround-Mum als ambivalente Sozialfigur der Corona-Pandemie, in: KWI-BLOG, [https://blog.kulturwissenschaften.de/vonsorgearbeit-und-gesellschaftlichen-scheinwerfern/], 11.01.2021

DOI: https://doi.org/10.37189/kwi-blog/20210111-0830 


\section{DuEPublico}

offen im Denken

\section{Duisburg-Essen Publications online}

Dieser Text wird über DuEPublico, dem Dokumenten- und Publikationsserver der Universität Duisburg-Essen, zur Verfügung gestellt. Die hier veröffentlichte Version der EPublikation kann von einer eventuell ebenfalls veröffentlichten Verlagsversion abweichen.

DOI: $\quad$ 10.37189/kwi-blog/20210111-0830

URN: urn:nbn:de:hbz:464-20210114-105946-2

Alle Rechte vorbehalten. 